\title{
Self-referenced multiplex CARS imaging with picosecond pulse generated supercontinuum by using second and third order nonlinearities
}

\author{
Sahar Wehbi ${ }^{1,2}$, Tigran Mansuryan ${ }^{1}$, Marc Fabert ${ }^{1}$, Alessandro Tonello ${ }^{1}$, Katarzyna Krupa ${ }^{3}$, Stefan \\ Wabnitz $^{4}$, Sébastien Vergnole ${ }^{2}$ and Vincent Couderc ${ }^{1}$ \\ 1. University of Limoges, XLIM, UMR CNRS 7252, 123 Avenue A. Thomas, 87060 Limoges, France \\ 2. ALPhANOV, Optics \& Lasers Technology Center, Institut d'optique d'Aquitaine, Rue François Mitterrand, 33400 Talence, France \\ 3. Institute of Physical Chemistry, Polish Academy of Sciences, 01-224 Warsaw, Poland \\ 4. DIET, Sapienza University of Rome Via Eudossiana 18, 00184 Rome, Italy
}

Among the various imaging methods, the multiplex Coherent Anti-Stokes Raman Scattering (M-CARS) technique has received a special attention from the researchers. Indeed, its capability to provide quickly, simultaneous information about various molecular vibrations is of high interest. Over the past years M-CARS microscopy has typically relied on using a supercontinuum, which is generated in photonics crystals fibers (PCFs) with the cubic nonlinearity. This technique requires an additional optical delay line to temporally synchronize the pump and the Stokes pulses [1]. Significant simplification could be introduced by exploiting other nonlinear processes that allows to generate ultra-broadband spectrum without temporal desynchronization between the pump and the supercontinuum pulses. In this work we demonstrate three direct, self-referenced M-CARS systems that do not require any delay line, by using different nonlinear components such as a PPLN crystal $\left(\chi^{(2)}\right.$ and $\left.\chi^{(3)}\right)$; a YAG:Nd crystal $\left(\chi^{(3)}\right)$ and a GRIN multimode fiber $\left(\chi^{(3)}\right)$ under the Kerr beam self-cleaning regime [2].

In our studies we used a laser source at $1030 \mathrm{~nm}$ with a tunable pulse duration: crystals were pumped by $3 \mathrm{ps}$ pulses, while the GRIN multimode fiber was pumped by 80 ps pulses. In the case of the crystals, because of the relatively short length (typically $20 \mathrm{~mm}$ to $30 \mathrm{~mm}$ ) and because of a strong nonlinearity, we minimize the relative temporal delays, thus keeping the supercontinuum synchronized with the pump beam. In the GRIN multimode fiber the synchronization between the pump and the Stokes waves resulted from the long temporal duration of the input pulses; moreover, the beam self-cleaning secured a level of residual pump strong enough to obtain the whole M-CARS signal. Supercontinuum spectra were controlled by varying the input beam polarization orientation and the power level.
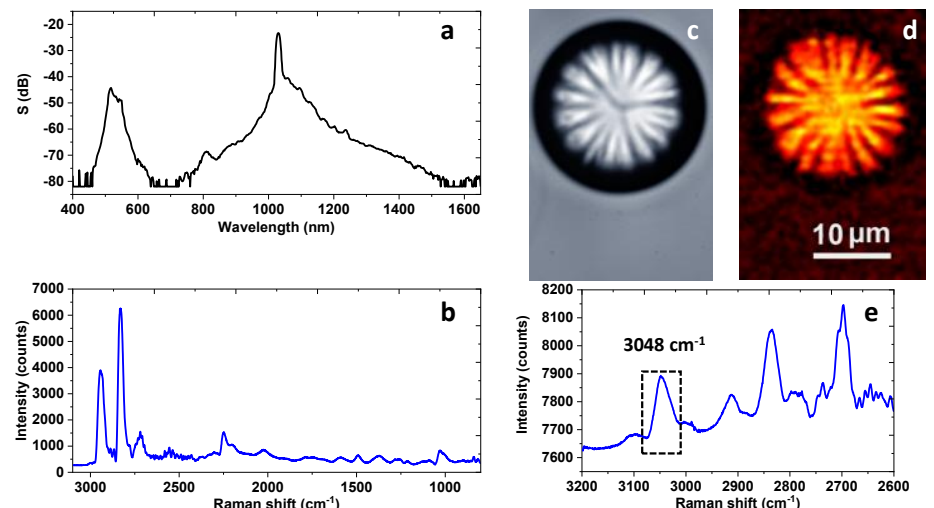

Fig. 1 Supercontinuum (a) generated in PPLN was used to obtain M-CARS spectrum of methanol (b). Bright-field image (c) of a polystyrene bead and the corresponding M-CARS image (d). Peak at $3048 \mathrm{~cm}^{-1}$ was selected in M-CARS spectrum of polystyrene (e), to have a M-CARS image of the polystyrene bead (d).

To validate our method, the generated supercontinuum was delivered without any optical delay line, through several spectral filters, into a microscope to analyze the different samples. Those filters helped to remove the visible spectrum and to narrow the pump spectrum. Fig. 1 shows an example of the supercontinuum, generated in a PPLN, by mixing the contribution of $\chi^{(2)}$ and $\chi^{(3)}$ nonlinearities. The control of the polarization orientation in the crystal allows to achieve an accurate supercontinuum shaping, which can further favors the emission in the $\mathrm{C}-\mathrm{H}$, or in the fingerprint regions. The M-CARS spectrum of methanol and the M-CARS image of polystyrene bead are shown in Fig.1. Additional results obtained by using cubic crystals and multimode fibers will also be presented.

\section{References}

[1] P. A. Champert, V. Couderc, A. Barthélémy, "1.5-2.0-/spl mu/m multiwatt continuum generation in dispersion-shifted fiber by use of highpower continuous-wave fiber source," IEEE PTL 16(11), 2445-2447 (2004).

[2] K. Krupa, A. Laburyère, A. A. Tonello, B. M. Shalaby, V. Couderc, "Polychromatic filament in quadratic media: spatial and spectral shaping of light in crystals," Optica 2(12), 1058-1064 (2015). 